\title{
A multi-discretization scheme for topology optimization based on the parameterized level set method
}

Peng $\mathrm{Wei}^{1,2,{ }^{*}}$, Yang $\mathrm{Liu}^{1}$, and Zuyu $\mathrm{Li}^{3}$

${ }^{1}$ School of Civil Engineering and Transportation, State Key Laboratory of Subtropical Building Science, South China University of Technology, 381 Wushan Rd, Tianhe, Guangzhou, PR China

${ }^{2}$ State Key Laboratory of Structural Analysis for Industrial Equipment, Dalian University of Technology, 2 Linggong Rd, Ganjingzi, Dalian, PR China

${ }^{3}$ Architecture and Civil Engineering Institute, Guangdong University of Petrochemical Technology, 139 Guandu 2nd Rd, Maoming, PR China

Received: 17 May 2019 / Accepted: 21 October 2019

\begin{abstract}
In the framework of the parameterized level set method, the structural analysis and topology representation can be implemented in a decoupling way. A parameterized level set function, typically, using radial basis functions (RBFs), is a linear combination of a set of prescribed RBFs and coefficients. Once the coefficients are determined, the theoretical level set function is determined. Exploiting this inherent property, we propose a multi-discretization method based on the parameterized level set method. In this approach, a coarse discretization is applied to do the structural analysis whereas another dense discretization is employed to represent the structure topology. As a result, both efficient analysis and high-resolution topological design are available. Note that the dense discretization only accounts for a more precise and smooth description of the theoretical level set function rather than introduce extra design freedom or incur interference to structural analysis or the optimization process. In other words, this decoupling way will not add to the computational burden of structural analysis or result in non-uniqueness of converged results for a particular analysis setting. Numerical examples in both two-dimension and three-dimension show effectiveness and applicability of the proposed method.
\end{abstract}

Keywords: Parameterized level set / multi-discretization / radial basis functions / topology optimization

\section{Introduction}

For the past decades, methods of topology optimization have achieved significant development and have been applied to a wide variety of industries. Basically, for certain constraints, topology optimization aims at finding the optimal material distribution and maximizing the performance of the resulting structure. Up to present, the most widely used topology optimization methods include: the density-based methods such as the Solid Isotropic Material with Penalization (SIMP) method [1,2], the homogenization method [3,4], and the evolutionary method [5]; the boundary evolution methods such as the phase field method [6,7], the level set method [8-12], the explicit interface represent method [13]; the methods with combinations of discrete components [14,15].

Generally, the implementation of topology optimization is driven by structural analysis, while the description of structure topology varies from voxels to topological

\footnotetext{
* e-mail: ctpwei@scut.edu.cn
}

description functions for different categories of methods. Despite different ways of topology description, a common goal for various optimization methods is to reduce computational cost while simultaneously maintaining high-resolution topology of structures. Conventionally, in the density-based framework, the density elements are directly mapped on analysis mesh and they are used for topology description at the same time, which accounts for limited topological resolution. To counter this issue, decoupling strategy of analysis discretization and topology description are widely investigated. Nguyen et al. [16] developed a multi-resolution approach, in which a coarser finite element mesh is applied for the structural analysis while a finer design variable mesh is used for the optimization and topology representation, to balance the efficiency and the high-resolution. Further improvement of this method with multiple discretization was introduced in [17]. Applications of this multi-resolution strategy for different problems can be found in [18-20]. Wang et al. [21] proposed a strategy that allows for adaptive highresolution of the topology. With the refined or coarsened 


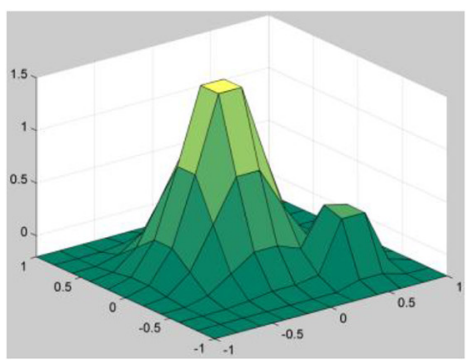

(a)

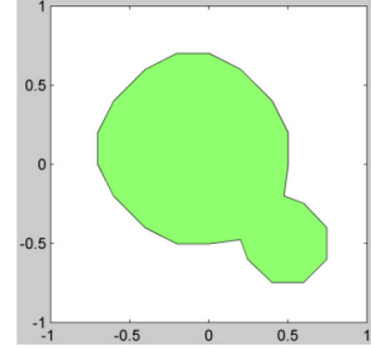

(b)

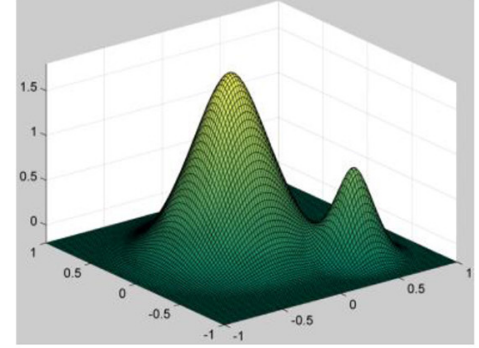

(c)

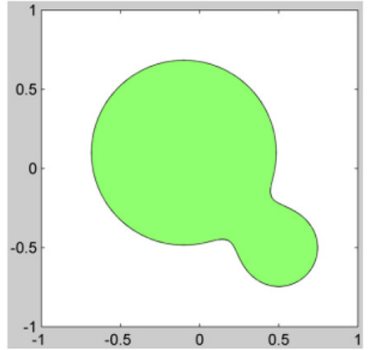

(d)

Fig. 1. Plots for the level set function and its zero-level set. (a) A coarse representation for the level set function. (b) A coarse representation for the zero-level set. (c) A refined representation for the level set function. (d) A refined representation for the zero-level set.

density point field of particular local regions, the topological resolution can be improved as well as the optimization efficiency. Groen et al. [22] used finite cell method (FCM) [23] to determine the potentials and limitations of high-order multi-resolution topology optimization. Typically, Gupta et al. [24] studied the upper bounds for the number of design variables per finite element which are used to enhance topological resolution without losing efficiency and involving non-uniqueness of optimization results. The decoupling strategy is efficient and straightforward. Researches mentioned above have reported success in higher resolution of structure topology at reasonable computation cost. Although above densitybased methods can alleviate the visually jagged boundaries to some extent, the intrinsic voxel description has not changed. In other words, the jagged issue will occur after scaling up of the topology. Besides, the introduction of extra design variables aimed at achieving higher resolution of topology will not enlarge the design space for a particular analysis setting but rather add to the sensitivity analysis burden.

In this study, the authors propose an efficient multidiscretization strategy in the parameterized level set framework to achieve smoother and more precise topology at reasonable computation cost, which is also a decoupling strategy. A straightforward coarse discretization based on first-order finite elements is employed to do the structural analysis, while a refined discretization is applied to represent the structure topology. In this approach, radial basis functions (RBFs) are implemented to parameterize the level set function, then it can be represented by a linear combination of a set of prescribed RBFs and coefficients, which are also the design variables in this framework. The RBFs knots are mapped on the finite element analysis mesh nodes and have the same number of mesh nodes. In this way, the design space is adequately exploited without introducing extra design variables or resulting in non-uniqueness of converged topology for a particular analysis setting. In addition, under the framework of parameterized level set, topological resolution is no longer a problem because the level set function has a clear analytical expression. This is totally different compared with voxels description because scaling will not change the display resolution. However, the representation of the topology is also discretization-dependent. A coarse discretization usually cannot capture more detailed features for a given theoretical level set function while a refined discretization can describe that more precisely. For example, for a given level set function $\phi$ :

$$
\begin{gathered}
\phi=2\left(\max \left(0,1-r_{1}\right)\right)^{4} \cdot\left(4 r_{1}+1\right) \\
+\left(\max \left(0,1-r_{2}\right)\right)^{4} \cdot\left(4 r_{2}+1\right)-0.2 \\
r_{1}=\sqrt{(x+0.1)^{2}+(y-0.1)^{2}} x, y \in[-1,1] \\
r_{2}=0.5 \sqrt{(x-0.5)^{2}+(y+0.1)^{2}} x, y \in[-1,1]
\end{gathered}
$$

where $x, y$ are coordinates. Figure $1 \mathrm{a}$ and $\mathrm{b}$ shows the plots of the given level set function and its zero-level set with a coarse discretization respectively. Figure 1c and d shows the plots of the given level set function and its zero-level set with a refined discretization respectively.

As can be seen, although there are no resolution issues for both coarse and refined representation using the explicit expression, refined discretization achieves smoother and more precise representation of the theoretical level set function. Herein, the refined discretization only accounts for a more precise and smooth description of the theoretical level set function rather than introduce extra design freedom or incur interference to structural analysis or the optimization process. Both the coarse representation and the refined representation are interpolated by the prescribed RBFs and their according coefficients. However, the refined representation does not have to be involved in the optimization process and it can be used as a postoptimization process based on obtained optimal design variables rather than some heuristic schemes. In other words, this decoupling way will not add to the computational burden of structural analysis while maintaining a smoother and more precise description. A disadvantage of this decoupling strategy would be that the different representations will usually lead to minor differences in material volume, which we believe would be an acceptable compromise.

The rest of this article is organized as follows: Section 2 gives a brief theoretical background of the RBFs parameterized level set-based topology optimization 


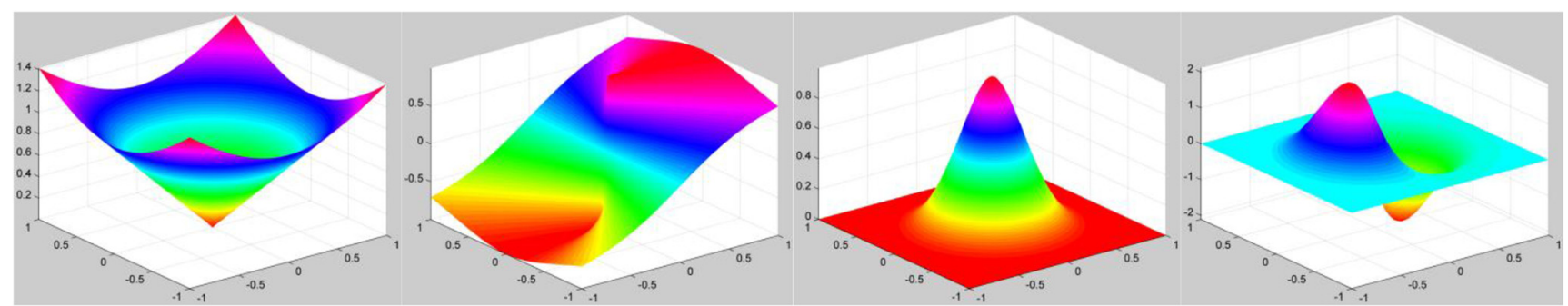

(a)

(b)

(c)

(d)

Fig. 2. RBFs plot. (a) shows an MQ spline and (b) gives its partial derivative in $x$ direction. (c) and (d) show the plots for a $C^{2}$-CSRBF and its partial derivative with respect to $x$ direction respectively.

method. Section 3 formulated a standard minimum compliance optimization problem. Followed by numerical examples in Section 4 and concluding remarks in Section 5.

\section{RBFs parameterized level set method}

The concept of parameterization is using a linear combination of a set of RBFs and coefficients to construct the level set function. The spatial coordinates of RBFs knots are prescribed before the optimization process. Once the coefficients are determined, the level set function is constructed regardless of the finite element (FE) analysis meshes. In this way, the level set function can be driven to optimal topology by updating the coefficients. There are two types of RBFs are commonly used according to the support radius: Globally Supported Radial Basis Functions (GSRBFs) and Compactly Supported Radial Basis Functions (CSRBFs). Equations (4) and (5) give the expressions of the Multi-quadrics (MQ) spline (GSRBF) [25] and the $C^{2}$ Wendland CSRBF [26] respectively:

$$
\begin{gathered}
g(r)=\sqrt{r^{2}+C^{2}} c \in \mathbb{R}^{1} \\
g(r)=(\max (0,1-r))^{4} \cdot(4 r+1)
\end{gathered}
$$

where $r$ is the support radius and $c$ is a parameter with a small positive value. For a two-dimensional case, Figure 2 displays the plots of an MQ spline and a $C^{2}$ CSRBF and their partial derivatives with respect to $x$ direction.

In the level set-based optimization model, the level set function $\phi(\mathbf{x})$ can be used to represent the design:

$$
\left\{\begin{array}{l}
\phi(\mathbf{x})>0 \forall \mathbf{x} \in \Omega \backslash \partial \Omega \\
\phi(\mathbf{x})=0 \forall \mathbf{x} \in \partial \Omega \\
\phi(\mathbf{x})<0 \forall \mathbf{x} \in D \backslash \Omega
\end{array}\right.
$$

where $\mathbf{x} \in \mathbb{R}^{2}$ or $\mathbb{R}^{3}$ is a point in the full design domain $D$ and $\partial \Omega$ denotes the boundary of the design $\Omega$. To update the level set function, the Hamilton-Jacobi equation, which is given in equation (7), is usually used in the conventional level set-based method $[10,12]$.

$$
\frac{\partial \boldsymbol{\phi}}{\partial t}-V_{n}|\nabla \boldsymbol{\phi}|=0
$$

where $V_{n}$ is the velocity vector along the normal direction of the boundary. After parameterization, the value of the level set function at an RBF knot $\overline{\mathbf{x}}$ can be calculated as:

$$
\phi(\overline{\mathbf{x}})=\sum_{j=1}^{m} \alpha_{j} g_{j}(\overline{\mathbf{x}})
$$

where $j$ indicates the $j$ th RBF knot, $m$ the total number of RBF knots, $\alpha_{j}$ the expansion coefficient of the $j$ th RBF and $g_{j}$ the $j$ th RBF. Since the coordinates of RBF knots and initial level set function value of the knots are given at first, the initial expansion coefficients $\alpha_{j}$ can be calculated through self-interpolation between RBF knots. The level set function in equation (8) can also be represented in a matrix form:

$$
\boldsymbol{\phi}=\mathbf{G} \boldsymbol{\alpha}
$$

where $\boldsymbol{\phi}=\left\{\phi\left(\overline{\mathrm{x}}_{1}\right), \phi\left(\overline{\mathrm{x}}_{2}\right), \cdots, \phi\left(\overline{\mathrm{x}}_{m}\right)\right\}^{T}$ and $\mathbf{G}$ is theoretically invertible. Here the pseudo time $t$ is introduced to represent the evolution of the level set function $\phi$ :

$$
\boldsymbol{\phi}(\overline{\mathbf{x}}, t)=\mathbf{G} \boldsymbol{\alpha}(t)
$$

Substituting equation (10) into the Hamilton-Jacobi equation defined in equation (7), we obtain:

$$
\mathbf{G} \frac{d \boldsymbol{\alpha}}{d t}-\mathbf{B}=0
$$

where:

$$
\mathbf{B}=V_{n}|\nabla \mathbf{G} \boldsymbol{\alpha}|
$$

The term $\mathbf{B}$ can also be transformed into the following form by introducing the normalization scheme in [27,28]:

$$
\mathbf{B}^{*}=\left\{\begin{array}{c}
\delta\left(\phi\left(\overline{\mathbf{x}}_{1}\right)\right) V_{n}\left(\overline{\mathbf{x}}_{1}\right) \\
\vdots \\
\delta\left(\phi\left(\overline{\mathbf{x}}_{m}\right)\right) V_{n}\left(\overline{\mathbf{x}}_{m}\right)
\end{array}\right\}
$$

where $\delta(\cdot)$ is an approximate Dirac $\delta$ function which can follow the form in [28]. According to equation (11), the following formulation can be used to update the expansion 


\section{- RBF knots and points for level set function}

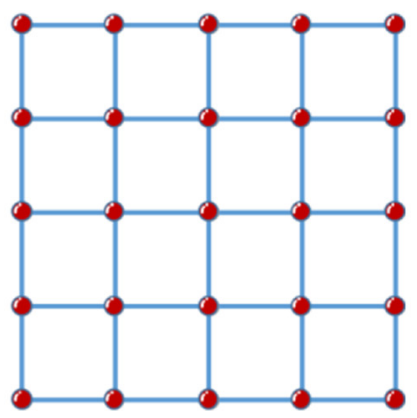

Mesh for FEA
Inserted points for level set function interpolation in refinement

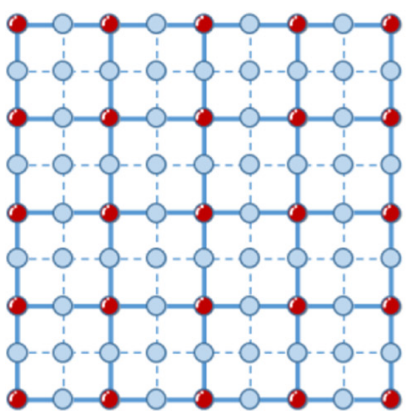

Mesh for refinement

Fig. 3. The refinement scheme by incerting more inpolateration points in the parameterized level set method for structured meshes.

coefficients $\boldsymbol{\alpha}(t)$ with the first order Euler method:

$$
\boldsymbol{\alpha}\left(t_{p+1}\right)=\boldsymbol{\alpha}\left(t_{p}\right)+\Delta t \mathbf{G}^{-1} \mathbf{B}^{*}\left(t_{p}\right)
$$

where $p$ indicates the $p$ th iterative step. In this method, because the value of the level set function is fully determined by the expansion coefficient $\boldsymbol{\alpha}$, it successfully avoids the difficulties of the updating scheme based on a spacial difference in unstructured meshes for the conventional level set method. The value of the level set function at any given point $\mathbf{x}$ in the design domain $D$ can be interpolated through the following formulation regardless of design domains (regular or irregular) and discretization (structured or unstructured mesh):

$$
\phi(\mathbf{x}, t)=\sum_{j=1}^{m} \alpha_{j} g_{j}(\mathbf{x}, t) .
$$

As shown in Figure 3, the values of the level set function on the inserted points can be directly calculated with equation (15) in a refined mesh as long as the coordinates of those points are given. Thus a smoother design can be obtained with the refined level set function. Here a structured mesh is used to illustrate its mechanism, but the interpolation with RBFs can be applied in unstructured meshes without further efforts as can be easily found in equation (15) and the numerical examples section later in this article.

Please note here only a basic and brief introduction of the RBFs-based parameterized level set method is provided to facilitate readership. The practical implementations also involve other numerical operations, thus the readers could refer to $[28,29]$ for more details.

\section{Topology optimization formulation}

In the topology optimization field, it is quite common to minimize the compliance of a structure. Herein the case of a statically loaded linear elastic structure subjected to a volume constraint is considered. The mathematical optimization model is formulated as follows:

$$
\operatorname{Min} J(\boldsymbol{\phi})=\int_{D}(\boldsymbol{\varepsilon}: \mathbf{E}: \boldsymbol{\varepsilon}) H(\boldsymbol{\phi}) d \Omega
$$

$$
\text { s.t. }\left\{\begin{array}{l}
\mathbf{K}(\boldsymbol{\phi}) \mathbf{U}=\mathbf{F} \\
G(\boldsymbol{\phi}) \leq V_{\max }
\end{array}\right.
$$

where $J(\phi)$ is the objective function, $\boldsymbol{\varepsilon}$ the strain tensor, $\mathbf{E}$ the elasticity tensor, $\mathbf{K}(\phi)$ the stiffness matrix, $\mathbf{U}$ the displacement field, $\mathbf{F}$ the external loads, $G(\phi)$ the total material usage of the structure and $V_{\max }$ the pre-specified upper limit of the allowable material usage in the design domain $D . H(\phi)$ is the Heaviside function as given in equation (17) which uses the value of the level set function to indicate the existence of the material at point $\mathbf{x}$ :

$$
H(\phi(\mathbf{x}))=\left\{\begin{array}{l}
1 \text { if } \phi(\mathbf{x}) \geq 0 \\
0 \text { if } \phi(\mathbf{x})<0
\end{array}\right.
$$

The sensitivity analysis is a key part of the topology optimization process, in which the material derivative is derived in each iteration step to calculate the evolution velocity field $V_{n}$. For a linear static compliance minimum problem, the normal velocity $V_{n}$ can be determined by the strain energy density [30] and a Lagrange multiplier $\lambda$ with the form given in equation (18).

$$
V_{n}=\boldsymbol{\varepsilon}: \mathbf{E}: \boldsymbol{\varepsilon}-\lambda
$$

Here the Lagrange multiplier $\lambda$ is applied to control the volume fraction to limit the material usage. It can be calculated with a bi-sectioning algorithm in each iteration. Please note in the level set-based optimization method, the volume fraction of the initial design generally can hardly meet the volume constraint. To avoid the instability caused by sudden changes in the total volume, the volume constraint is relaxed and transformed to the following form in the first $n_{R}$ iterations [28]:

$$
G^{k}=\int_{D} H(\boldsymbol{\phi}) d \Omega-\left[V_{0}-\left(V_{0}-V_{\max }\right) \frac{k}{n_{R}}\right] k \leq n_{R}
$$

where $k$ indicates the $k$ th iteration step, and $V_{0}$ is the initial volume.

The optimization takes an iterative way by applying the steepest descent method. The expansion coefficients $\boldsymbol{\alpha}(t)$ are directly updated with equation (14) until the 


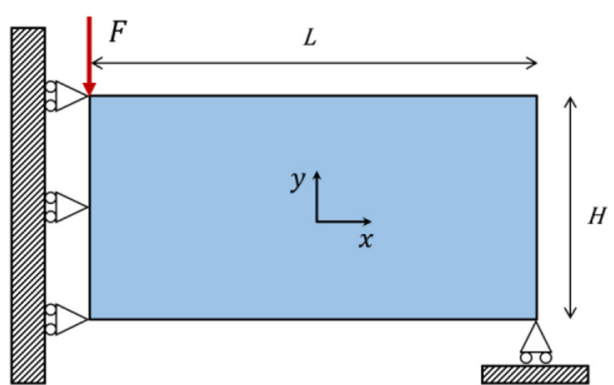

(a). The MBB beam problem.

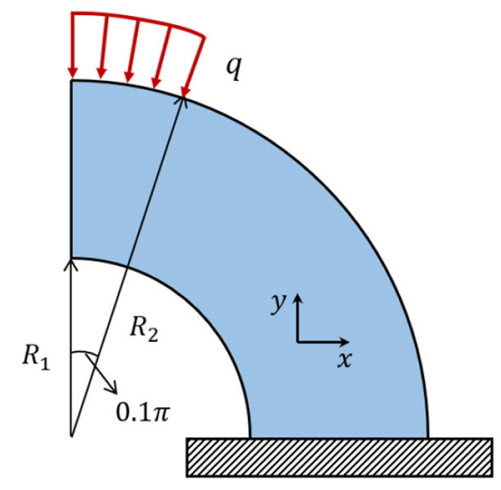

(c). The quarter ring problem.

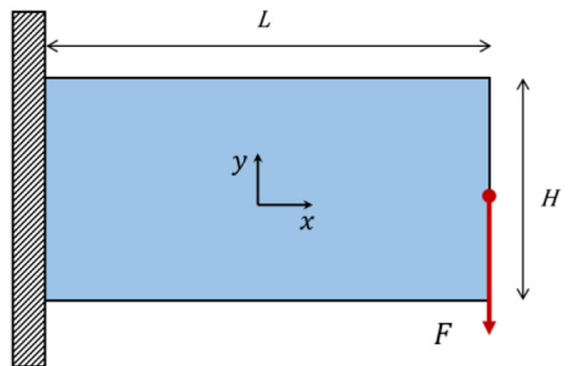

(b). The cantilever beam problem.

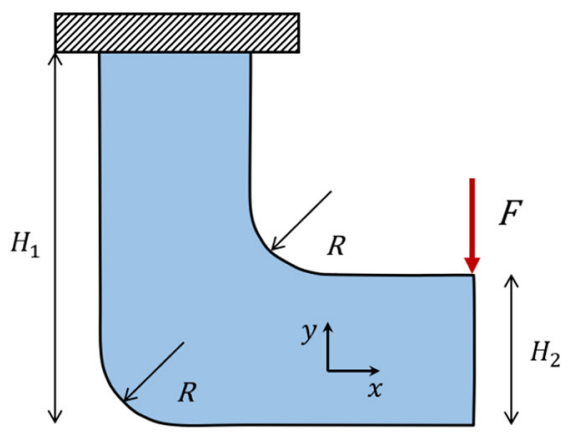

(d). The L-shaped beam problem.

Fig. 4. Definition of the design domains and the boundary conditions for the $2 \mathrm{D}$ problems.

optimality criterion or the maximum iterative step number is reached. The values of the level set function on RBF knots and FE nodes can then be attained through equation (15) to get the final optimal design. To obtain a refined representation of the design, the level set function values can be calculated on the refined grids with equation (15) to interpolate the zero-level set as illustrated in the following numerical examples.

\section{Numerical examples}

In this section, several numerical cases are discussed to illustrate the effectiveness of the proposed algorithm. Here we first introduce some numerical manipulations and basic parameter settings of the algorithm. The initial designs for all numerical examples are given as full material structures. The capacity of nucleation of the parameterized level set method can ensure the complex topological design solutions. Practical material properties are applied that Young's modulus and Poisson's ratio of solid material are $E=2.1 \times 10^{11} \mathrm{~Pa}$ and $v=0.3$ respectively. To avoid singularity, Young's modulus of weak material is set as $E_{\min }=10^{-4} E$.

\subsection{Numerical examples in two-dimension}

Four 2D problems are tested to show the effect of the multidiscretization scheme, i.e. the $\mathrm{MBB}$ beam problem, the cantilever beam problem, the quarter ring problem, and the
L-shaped beam problem. Fig. 4 shows the design domains and the boundary conditions of the four optimization problems. The MBB problem is subject to a downward point load at the upper left corner and the design domain meshes with $32 \times 16$ regular Q4 elements for analysis. Only half of the MBB beam is calculated because of the symmetry property. The length-scales of $L$ and $H$ are given at $2 d$ and $d$, respectively, where $d$ is a unit length. The refined discretization is chosen at a $120 \times 60$ mesh for topology representation. In the case of cantilever beam, the design domain is fixed at the left end, and a downward load is applied at the middle point of the right end. The lengthscales, analysis mesh and representative mesh are the same as the MBB problem. The quarter ring model is fixed at the bottom end and the uniform distributive load is applied along the radial direction. $R_{1}$ and $R_{2}$ are set to $d$ and $2 d$ respectively. The analysis mesh is discretized to 774 irregular quadrilateral elements and the representative mesh consists of 6069 interpolation points. The L-shaped beam is fixed at the upper end and a downward load is applied at the upper corner of the right end. $R, H_{1}$ and $H_{2}$ are set to $d, 5 d$ and $2 d$, respectively. The coarse analysis mesh consists of 851 irregular quadrilateral elements while the representative mesh consists of 6232 interpolation points. For all optimization problems, the allowable material volume fraction is set to $50 \%$.

Solutions of the tested 2D problems are displayed in Table 1 . The second column shows the converged solutions optimized and represented with coarse meshes. The third column shows the converged solutions optimized with 
Table 1. Comparison of several 2D problems with coarse and refined representation.

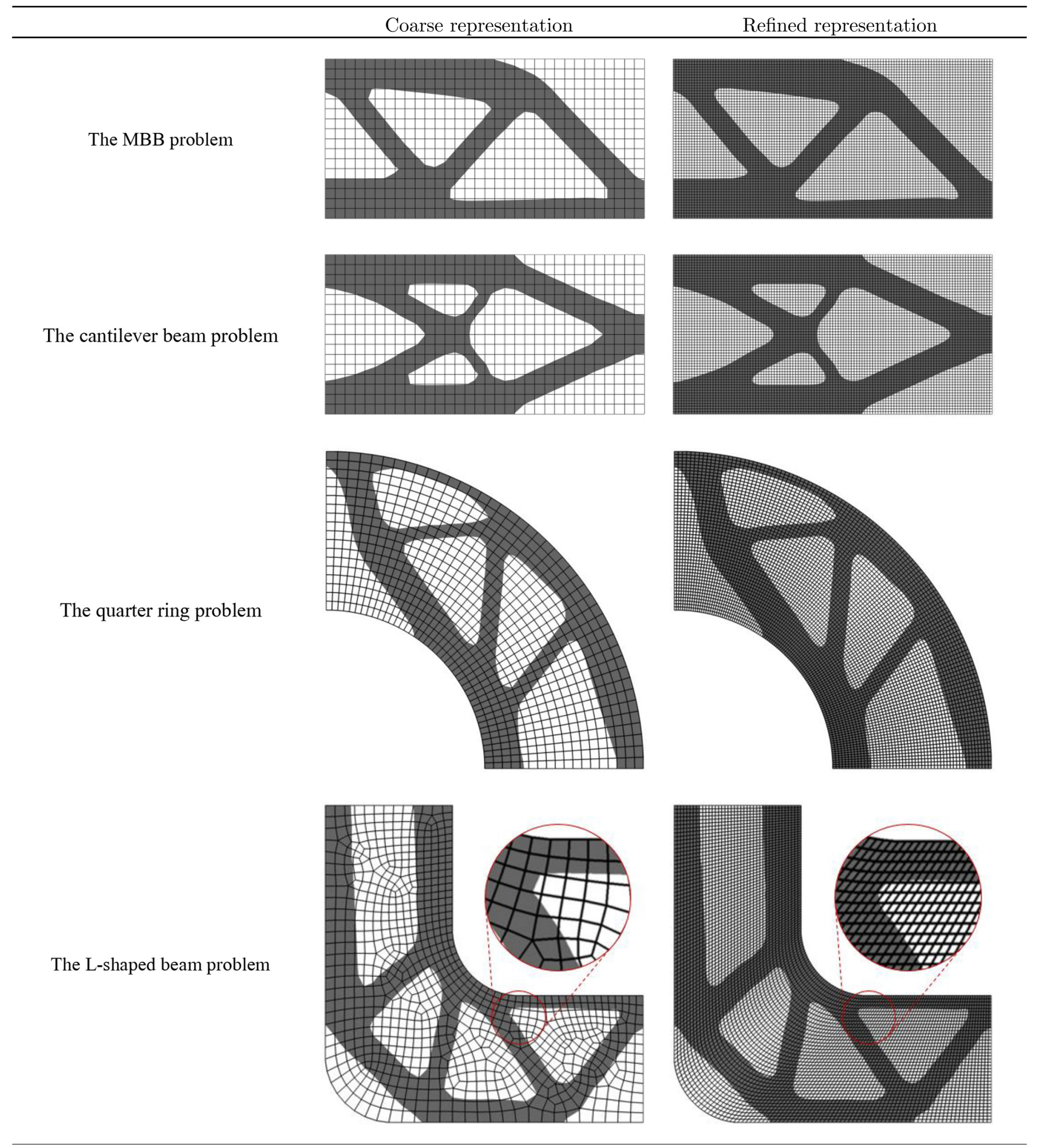

coarse analysis meshes but represented with refined meshes. As can be seen, compared with the coarse representation, the refined representation achieves smoother boundaries of the structures and it is a more precise description of the theoretical level set function since the two types of description are implemented for the same theoretical level set function that is fitted by the same prescribed RBF and the same optimized expansion coefficients. The refined mesh has many more interpolation points, which can help capture more detailed features of the structure. Instead of 


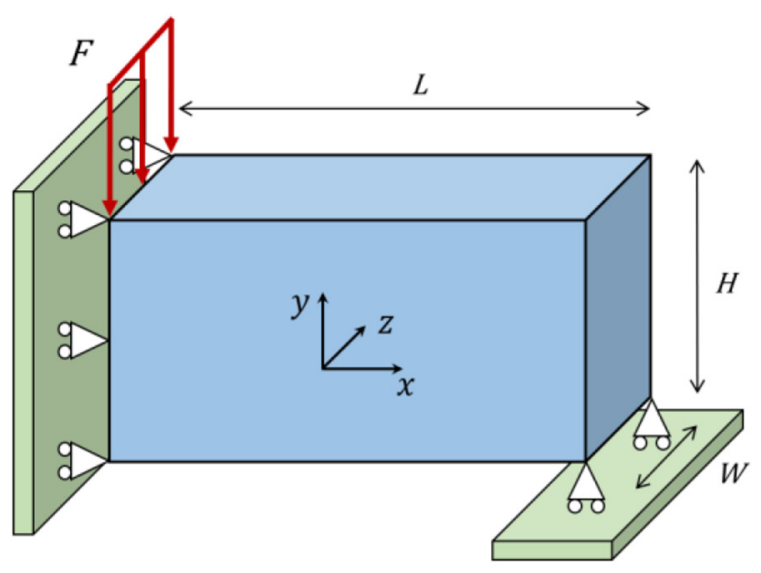

(a). The 3D MBB problem.

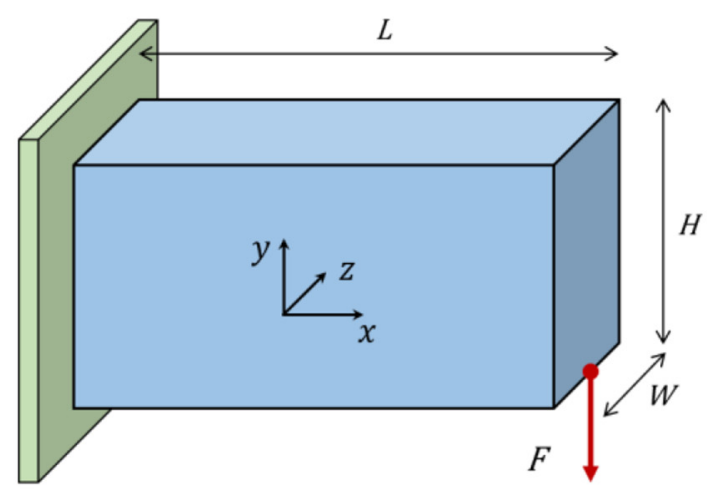

(b). The $3 \mathrm{D}$ cantilever beam problem.

Fig. 5. Definition of design domain and boundary conditions for the tested $3 \mathrm{D}$ problems.

Table 2. Comparison of the MBB problem with coarse and refined representation.

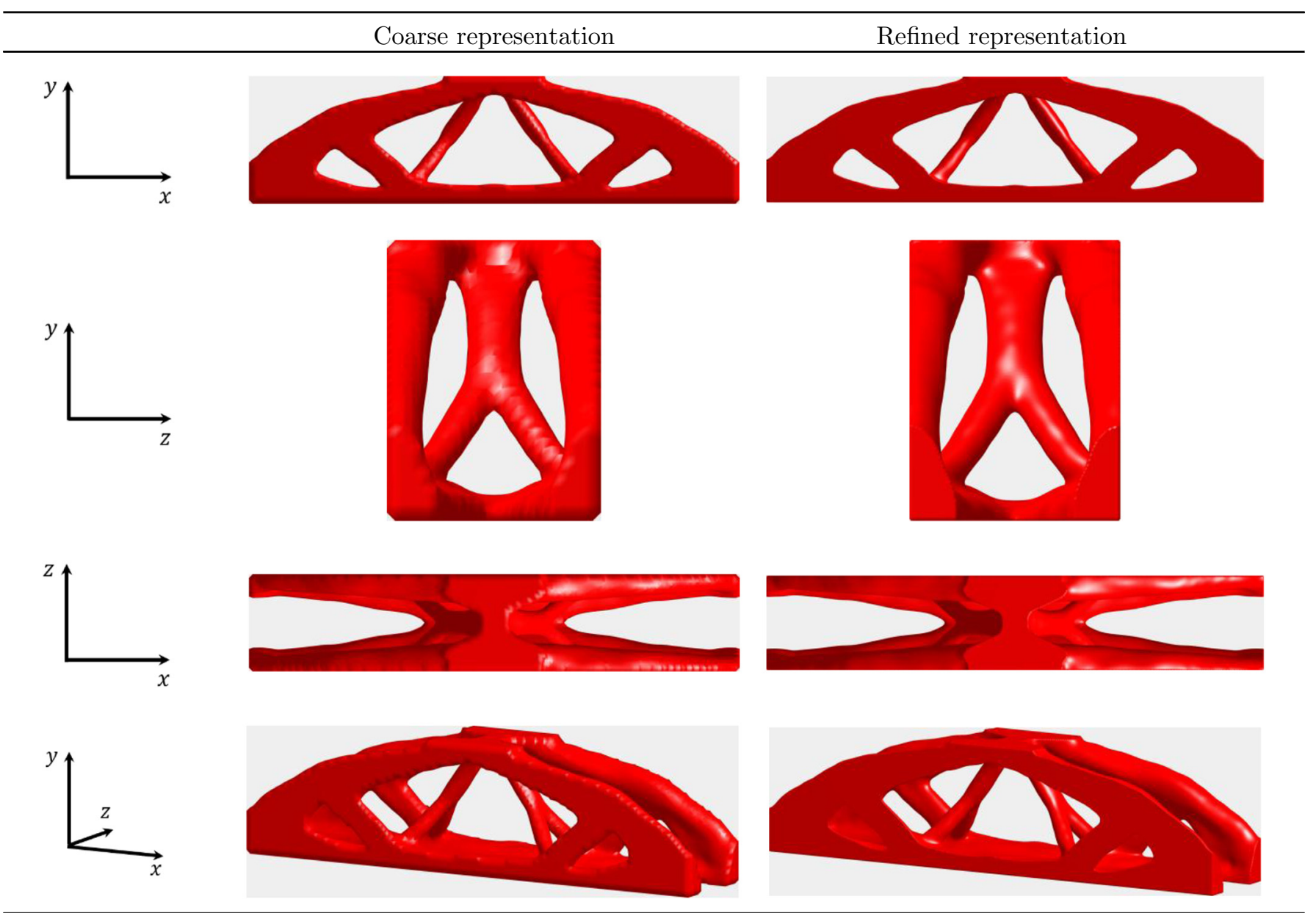

sharp change around boundary corners, structures show rounder features with the refined representation. Usually, the two types of structure descriptions can cause a difference in material volume, however, the volume variation between the coarse representation and the refined representation can be considered as the reduced error that can be negligible.
Herein, the refined mesh only accounts for more precise representation of the optimized result and it is not involved in the structural analysis. The FE analysis is performed on the coarse mesh and the RBF knot is coupled with the coarse analysis mesh node. As a result, the design space is not further exploited. 
Table 3. Comparison of the cantilever beam problem with coarse and refined representation.

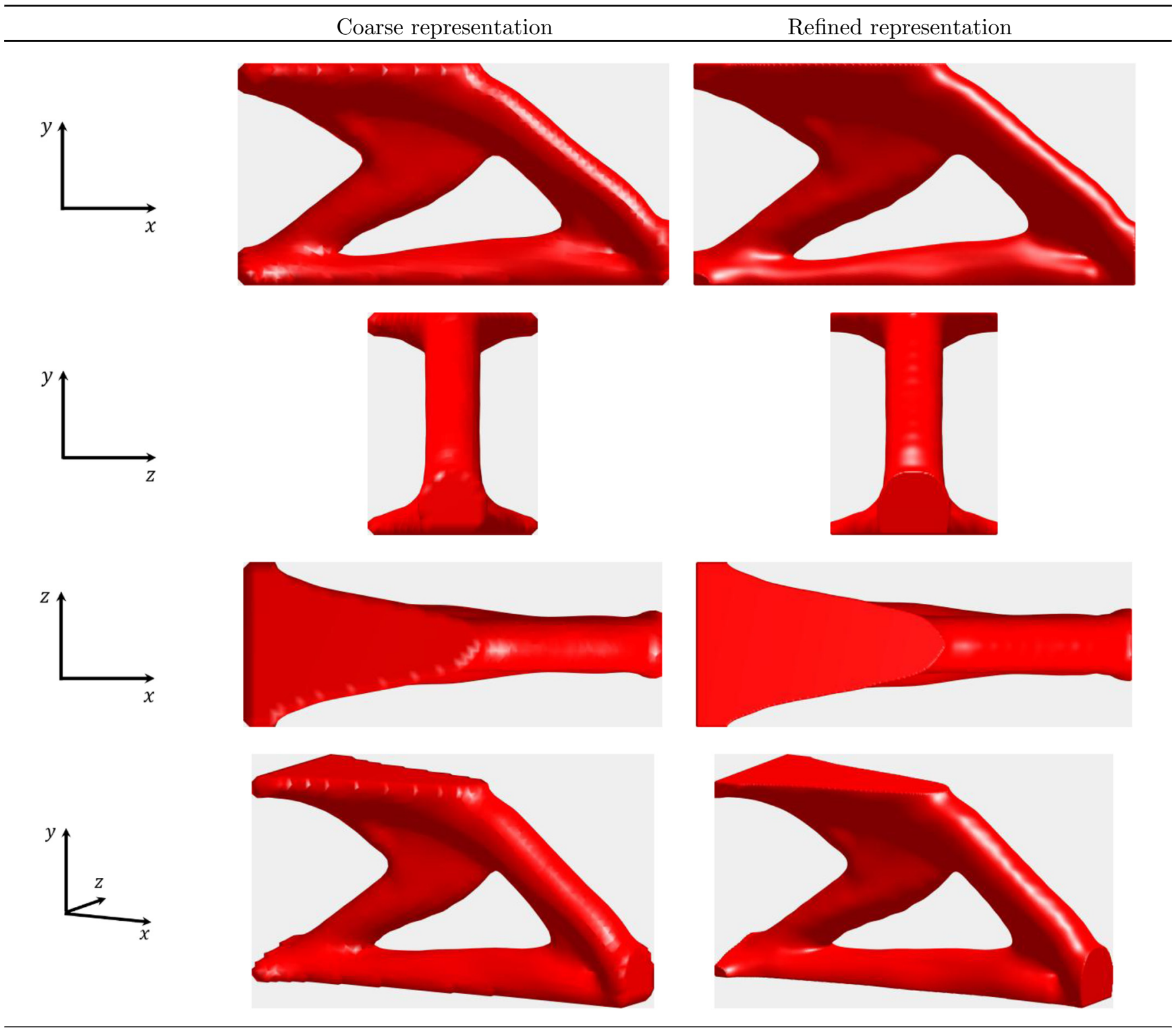

\subsection{Numerical examples in three-dimension}

Two 3D problems are tested to show the effect of the multidiscretization scheme. Figure 5 shows the definition of the design domain and boundary conditions of each of the two problems. In the case of the 3D MBB problem, taking the symmetry of the structure into account, only the right half of the structure is considered as the design domain. The $x$-direction displacement at left end of the MBB beam is constrained, and at the ends of the bottom right boundary of the beam, the $z$-direction displacement is constrained. The downward distributive load is applied at the upper left boundary of the beam. The length-scales of $L, H, W$ are given at $8 d, 4 d, 3 d$ respectively. The analysis mesh is discretized with $32 \times 16 \times 12 \mathrm{~B} 8$ elements in $x, y, z$ direction respectively. The refined representation is chosen at a $160 \times 80 \times 60$ mesh. In the case of cantilever beam, the design domain is fixed at the left end, and a downward point load is applied in the middle of the bottom right end. The length-scales, analysis mesh and representative mesh are the same as the MBB problem.

Solutions for the tested MBB problems and cantilever beam problem with different view perspectives are displayed in Tables 2 and 3 respectively. The second column of each of the two tables shows the converged solutions optimized and represented with coarse meshes. The third column shows the converged solutions optimized with coarse analysis meshes but represented with refined meshes. Similarly, compared with the coarse representation, the refined representation achieves smoother surfaces 
of the structures as a result of a more precise description of the theoretical level set function. Also, due to more interpolation points, more detailed features of the structure are captured.

\section{Conclusions and future works}

In this paper, we discuss a multi-discretization scheme based on parameterized level set. A coarse discretization is used to do the FE analysis while a refined discretization is used to represent the topology. As a result, both efficient analysis and smoother and more precise topology are available. In the parameterized level set framework, the refined representation can be used as an inherent tool of post-process since it is very efficient to implement as long as the optimized expansion coefficients of the RBF are determined, and it will not introduce non-uniqueness since it is a description of the same theoretical level set function. However, the limitation of the refined discretization is also quite straightforward that it is not involved in the optimization process and no further design space is exploited. The multi-discretization scheme can be further advanced to development where the analysis mesh, the design variable mesh, and the representation mesh are decoupled with each other. By employing higher-order shape functions, more coarse mesh can be used for analysis with accurate structural responses. By choosing an appropriate design variable mesh, the design space for a particular analysis setting can be adequately exploited. By applying a refined mesh, the topology representation can be precisely described. This compelling strategy will be discussed in future works.

The authors gratefully acknowledge the financial support provided by the National Natural Science Foundation of China under Grant No. 11372004 and State Key Laboratory of Structural Analysis for Industrial Equipment under Grant No. GZ18109.

\section{References}

1. G.I.N. Rozvany, M. Zhou, T. Birker, Generalized shape optimization without homogenization, Struct. Optim. 4, 250-252 (1992)

2. G.I.N. Rozvany, M.P. Bends $\phi$ E, U. Kirsch, Layout Optimization of Structures, Appl. Mech. Rev. 48, 41-119 (1995)

3. M.P. Bendsøe, N. Kikuchi, Generating optimal topologies in structural design using a homogenization method, Comput. Methods Appl. Mech. Eng. 71, 197-224 (1988)

4. J. Zhang, W.H. Zhang, J.H. Zhu, L. Xia, Integrated layout design of multi-component systems using XFEM and analytical sensitivity analysis, Comput. Methods Appl. Mech. Eng. 245-246, 75-89 (2012)

5. Y.M. Xie, Z.H. Zuo, X.D. Huang, J.H. Rong, Convergence of topological patterns of optimal periodic structures under multiple scales, Struct. Multidiscipl. Optim. 46, 41-50 (2012)

6. A. Takezawa, S. Nishiwaki, M. Kitamura, Shape and topology optimization based on the phase field method and sensitivity analysis, J. Comput. Phys. 229, 2697-2718 (2010)
7. T. Yamada, K. Izui, S. Nishiwaki, A. Takezawa, A topology optimization method based on the level set method incorporating a fictitious interface energy, Comput. Methods Appl. Mech. Eng. 199, 2876-2891 (2010)

8. S.J. Osher, F. Santosa, Level set methods for optimization problems involving geometry and constraints I. Frequencies of a two-density inhomogeneous drum, J. Comput. Phys. 171, 272-288 (2001)

9. J.A. Sethian, A. Wiegmann, Structural Boundary Design via Level Set and Immersed Interface Methods is, J. Comput. Phys. 163, 489-528 (2000)

10. M.Y. Wang, X.M. Wang, D.M. Guo, A level set method for structural topology optimization, Comput. Methods Appl. Mech. Eng. 192, 227-246 (2003)

11. M.Y. Wang, S. Zhou, Color level sets: a multi-phase method for structural topology optimization with multiple materials, Comput. Methods Appl. Mech. Eng. 193, 469-496 (2004)

12. G. Allaire, F. Jouve, A.M. Toader, Structural optimization using sensitivity analysis and a level-set method, J. Comput. Phys. 194, 363-393 (2004)

13. A.N. Christiansen, M. Nobel-Jørgensen, N. Aage, O. Sigmund, J.A. Bærentzen, Topology optimization using an explicit interface representation, Struct. Multidiscipl. Optim. 49, 387-399 (2013)

14. P. Wei, H. Ma, M.Y. Wang, The stiffness spreading method for layout optimization of truss structures, Struct. Multidiscip. Optim. 49, 667-682 (2014)

15. X. Guo, W. Zhang, W. Zhong, Doing topology optimization explicitly and geometrically-A new moving morphable components based framework, J. Appl. Mech. 81, 081009 (2014)

16. T.H. Nguyen, G.H. Paulino, J. Song, C.H. Le, A computational paradigm for multiresolution topology optimization (MTOP), Struct. Multidiscipl. Optim. 41, 525-539 (2009)

17. T.H. Nguyen, G.H. Paulino, J.H. Song, C.H. Le, Improving multiresolution topology optimization via multiple discretizations, Int. J. Numer. Methods Eng. 92, 507-530 (2012)

18. E.T. Filipov, J. Chun, G.H. Paulino, J. Song, Polygonal multiresolution topology optimization (PolyMTOP) for structural dynamics, Struct. Multidiscipl. Optim. 53, 673-694 (2015)

19. J. Park, A. Sutradhar, A multi-resolution method for 3D multi-material topology optimization, Comput. Methods Appl. Mech. Eng. 285, 571-586 (2015)

20. Q.X. Lieu, J. Lee, A multi-resolution approach for multimaterial topology optimization based on isogeometric analysis, Comput. Methods Appl. Mech. Eng. 323, 272302 (2017)

21. Y. Wang, Z. Kang, Q. He, An adaptive refinement approach for topology optimization based on separated density field description, Comput. Struct. 117, 10-22 (2013)

22. J.P. Groen, M. Langelaar, O. Sigmund, M. Ruess, Higherorder multi-resolution topology optimization using the finite cell method, Int. J. Numer. Methods Eng. 110, 903-920 (2017)

23. D. Schillinger, M. Ruess, The Finite Cell Method: A Review in the Context of Higher-Order Structural Analysis of CAD and Image-Based Geometric Models, Arch. Comput. Methods Eng. 22, 391-455 (2014)

24. D.K. Gupta, G.J. van der Veen, A.M. Aragon, M. Langelaar, F. van Keulen, Bounds for decoupled design and analysis discretizations in topology optimization, Int. J. Numer. Methods Eng. 111, 88-100 (2017) 
25. R.L. Hardy, Multiquadric equations of topography and other irregular surfaces, J. Geophys. Res. 76, 1905-1915 (1971)

26. H. Wendland, Piecewise polynomial, positive definite and compactly supported radial functions of minimal degree, Adv. Comput. Math. 4, 389-396 (1995)

27. X.H. Xie, M. Mirmehdi, Radial basis function based level set interpolation and evolution for deformable modelling, Image Vis. Comput. 29, 167-177 (2011)
28. P. Wei, Z.Y. Li, X.P. Li, M.Y. Wang, An 88-line MATLAB code for the parameterized level set method based topology optimization using radial basis functions, Struct. Multidiscipl. Optim. 58, 831-849 (2018)

29. S.Y. Wang, M.Y. Wang, Radial basis functions and level set method for structural topology optimization. Int. J. Numer. Methods Eng. 65, 2060-2090 (2006)

30. K.K. Choi, N.H. Kim, Structural Sensitivity Analysis and Optimization 1, Mech. Eng. 8, 189-194 (2006)

Cite this article as: Peng Wei, Yang Liu, Zuyu Li, A multi-discretization scheme for topology optimization based on a parameterized level set method, Int. J. Simul. Multidisci. Des. Optim. 11, 3 (2020) 